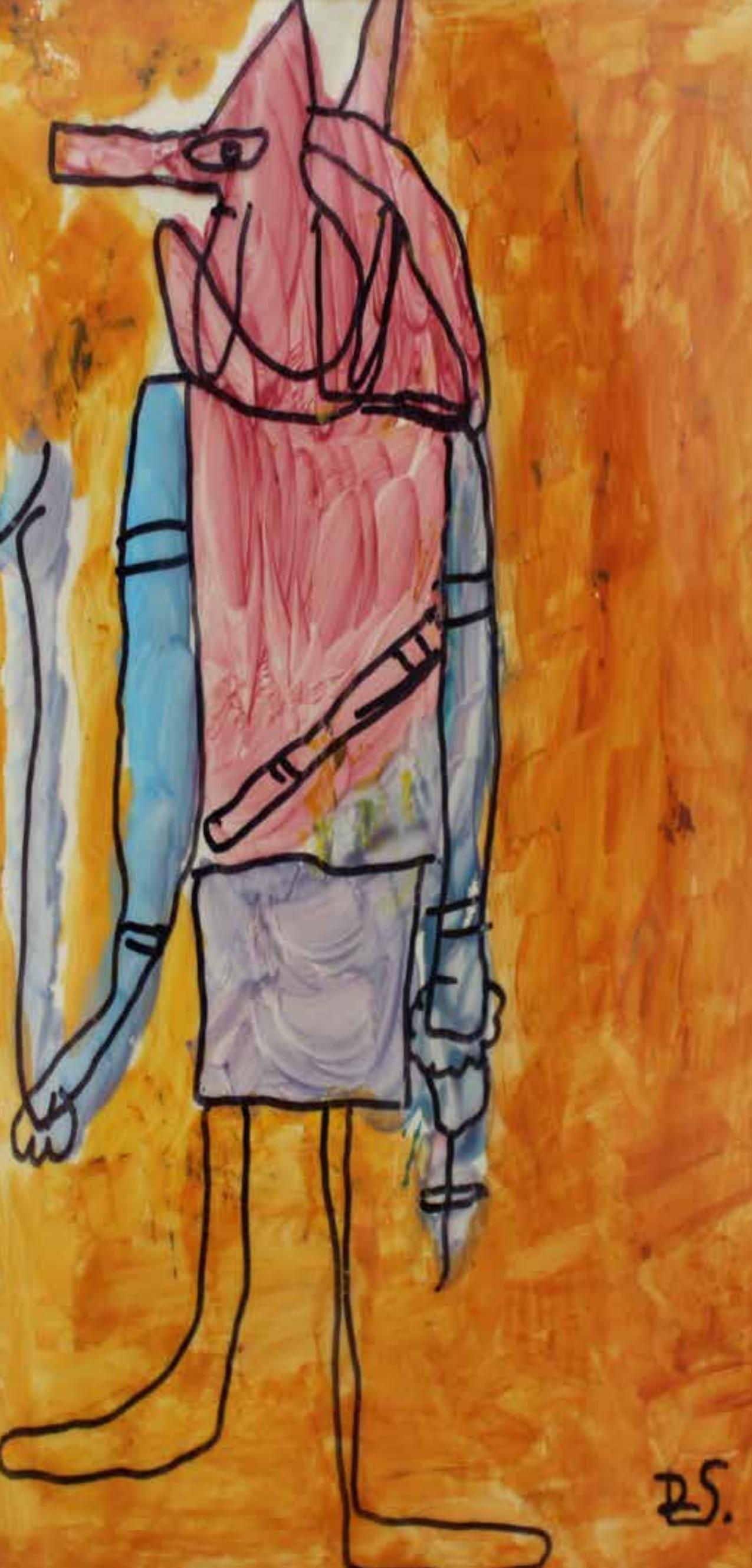

Marivel Montes Rotela

Fonoaudióloga. Magíster en Educación Universidad de Córdoba SUE Caribe. marivel.montes@unisucre.edu.co

Ivon Patricia Quessep Tapias

Fonoaudióloga, Especialista en Investigación aplicada a la Educación,

Corporación universitaria del Caribe CECAR ivon_quessep_7@hotmail.com

Lizbeth María Redondo Martínez,

Fonoaudióloga, Especialista en Investigación aplicada a la Educación, Corporación universitaria del Caribe CECAR Email: lizo811@hotmail.com

Karina Sofía Lastre Meza

Fonoaudióloga, candidata a Magister Universidad Internacional de la Rioja. sofilastre-15@hotmail.com

\title{
Diego Suarez
}

Discapacidad Intelectual

"HATSHEOSUT"
Vinilo sobre acetatos




\section{CARACTERIZACIÓN DEL LENGUAJE ORAL DE LA POBLACIÓN INDÍGENA ZENÚ, SUCRE Characterization of oral language of the indigenous population Zenú, Sucre}

Fecha de recepción: 21 de febrero de 2013 - Fecha de aprobación: 24 de abril de 2013

\section{RESUMEN}

Los Zenúes son un pueblo amerindio que hablaba la lengua Guajiba o Guamacó, pero en la actualidad hacen uso exclusivo del español, aunque conservan producciones que evidencian rasgos fonéticos y semánticos específicos poco conocidos. El desconocimiento de su filiación lingüística y el de sus particularidades motivó el presente trabajo, el cual muestra la caracterización del lenguaje oral de la población del resguardo indígena Zenú de San Andrés de Sotavento de Sucre, buscando reconocer las diferencias en contenido, forma y uso que permitirían rescatar su riqueza lingüística y el acervo cultural de ello para la costa Caribe. El estudio fue descriptivo y de corte transversal, incluyó técnicas de recolección mixtas aplicadas a 381 indígenas del resguardo mencionado. Los resultados evidenciaron procesos de simplificación en fonemas y sílabas, omisión de elementos sintácticos, preferencia de verbos en presente y pasado, cambios en referentes semánticos, léxico receptivo limitado y básico pero coherente, presencia de sobreextensiones en la significación y argumentación, características conversacionales pasivas para mantener, esperar y ceder turnos y empleo de gestos naturales como mediadores de la interacción. Se concluyó que existen particularidades fonológicas atribuidas al contexto socio cultural de la población; a nivel morfosintáctico predomina el empleo de estructuras gramaticales simples y complejas de tipos yuxtapuestas y coordinadas. En los niveles semántico y pragmático se evidenció reconocimiento de conceptos, empleo de vocablos contextualizados; introversión y escasas destrezas conversacionales que dan cuenta de pasividad en la interacción.

PALABRAS CLAVE Indígena, lenguaje, semántica, fonética, fonología, pragmática, morfosintaxis

\section{ABSTRACT}

The Zenúes are an Amerindian people who spoke the Guamacó language, but today they use the Spanish, although they retain productions that demonstrate specific and little known semantic and phonetic features. The neglect of their linguistic affiliation and their particularities motivated the research team to develop this work, which presents the characterization of oral language of the population of the Zenú indigenous reservation of San Andrés de Sotavento in the department of Sucre, seeking recognition of the differences in content, form and use, that would allow rescue its rich linguistic and cultural heritage of this for the Caribbean Coast. The study was descriptive and cross-sectional; it included mixed collection techniques which were applied to 381 indigenous people belonging to the reservation mentioned above. The results showed simplification processes in phonemes and syllables, omission of grammatical elements and preference of present and past verbs, changes in semantic referents, limited and basic receptive vocabulary, nevertheless, it is consistent; they also showed presence of overextension in the significance and argumentation, passive conversational features to keep, to wait and to give up the turn and using of natural gestures as mediator of the interaction. In conclusion, this study reveals that there are phonological particularities attributed to socio-cultural population context; it shows predominant use of simple and complex grammatical structures of juxtaposed and coordinated type in the morphosyntactic level; as well as recognition of concepts and use of contextualized words; and at pragmatic level, most people are characterized as introverted, they have poor conversational skills, so that, they are passive actors in the conversation.

KEY WORDS

Indigenous, Language, Semantics, Phonetics, Syntax, Pragmatics, morphosyntax. 
de la comunidad Zenú, como un tributo a su etnia, raza y legado histórico cultural del territorio colombiano. Así mismo, para el interés particular de la Fonoaudiología este proyecto resultó ser indispensable, dado que amplía el conocimiento en lo relacionado con su objeto de estudio (comunicación), donde el Lenguaje se constituye un campo de acción significativo dentro del contexto multicultural.

Teóricamente los postulados de Acosta y Moreno (1999), Gallardo y Gallego (2000) y Acosta et al. (2002) sirvieron de base para enmarcar la metodología y los criterios de análisis de la evaluación cualitativa del lenguaje desde la perspectiva fonoaudiológica. De igual forma, se adoptaron las conceptualizaciones de autores como Paternina (2002), Aleza y Enguita (2010), Trillos (2001) en lo concerniente a las bases teóricas de la dimensión de Forma; Vygotsky (1995), Bernstein y Bruner (citados por Gallardo y Gallego, 2000), Oleron (1985), Luria (1985) en la dimensión de Contenido; y Haverkate (1997), Hosfstadt (2005), Siebold (2008) en la de Uso.

\section{MÉTODO}

Como comunidad indígena, los Zenú, Zenúes o Sinú, pueblo amerindio cuya lengua desapareció y que actualmente habla español, han sido objeto de pocos estudios, entre ellos el realizado por Turbay y Jaramillo (2000), quienes afirman que el lenguaje usado por la población se caracteriza por conservar la " $h$ " aspirada del español antiguo, por ejemplo no dicen "hilo" sino "jilo" y que los ancianos sustituyen el fonema /d/ por /r/; de igual forma, recopilaron un vocabulario de aproximadamente 47 palabras que aún se conservan de su lengua antigua y que se caracterizan por la alta frecuencia del fonema $/ \mathrm{ch} /$.

Esto coincide con lo expuesto por Paternina (2002) quien recopiló varios términos que dan idea de la abundancia cultural dentro del cabildo de San Andrés de Sotavento, donde claramente se aprecia la caracterización fonológica de la " $\mathrm{h}$ " aspirada y la sustitución de /d/ por /r/, tales como: "Coddoba por Córdoba", "jamaca por hamaca", "jilo por hilo", "joccón por horcón", "jommiga por hormiga”, "jacha por hacha", "vedde por verde", "jabacca por abarca", entre otras.

Todas estas particularidades lingüísticas de la comunidad indígena son características inherentes a su expresión, que resultan de los rasgos culturales que los identifican (Acosta, Moreno, Ramos, Adelia, y Espino, 2002). Sin embargo, son escasos los referentes que describen las dimensiones de forma, contenido y uso del lenguaje de la población, lo que motivó a preguntarse ¿Cuáles son las características del lenguaje oral de los habitantes del resguardo indígena de San Andrés de Sotavento de Sucre?

Para dar respuesta a dicho interrogante se desarrollaron objetivos encaminados a identificar los rasgos particulares a nivel fonético-fonológico y morfosintáctico; describir las características del nivel semántico y determinar las particularidades del nivel pragmático que utiliza la población objeto de estudio. Por lo tanto, se constituyó en un estudio importante para el conocimiento de la cultura Sucreña propendiendo por el beneficio en la salud, la educación y la participación social, resaltando a su vez, el valor cultural y lingüístico

Estudio de tipo descriptivo de corte transversal, porque tuvo por objeto la representación de una situación o evento; de igual forma, se estudió en un mismo momento distintos individuos, los cuales representan diferentes etapas de desarrollo (Hernández, Fernández y Baptista, 2003). Para llevarlo a cabo se emplearon técnicas de recolección de la información de tipo cualitativo y cuantitativo con el fin de lograr una visión más integral del fenómeno de estudio.

En este estudio participaron indígenas adscritos al cabildo de San Andrés de Sotavento del departamento de Sucre (Municipios de San Onofre, Tolú, Colosó, Sincelejo, Toluviejo y Sampués), contando con una población de 61.644 sujetos, de la cual se seleccionó una muestra no probabilística por conveniencia de 381 indígenas, teniendo en cuenta la disponibilidad y accesibilidad de la población, cuyo tamaño muestral fue calculado mediante el paquete estadístico STATCALD- EPIINFO versión 7, con una frecuencia esperada del 50\%, un límite de confianza del 5\% y un 95\% de confianza total; repartiéndose la misma según la siguiente relación estadística que se observa en la Tabla 1. Para la selección de la muestra se establecieron como criterios de inclusión tener una edad entre 7- 65 años, pertenecer al resguardo indígena de San Andrés de Sotavento (Sucre); se excluyeron individuos con sordera y ceguera clínicamente diagnosticadas, alteraciones anatomofuncionales visibles a nivel de órganos fonoarticuladores, dificultades en la articulación o elocución del lenguaje y antecedentes neurológicos evidentes.

Se utilizaron técnicas de recolección de la información de tipo cuantitativo y cualitativo, las cuales se validaron mediante prueba piloto aplicada a una población de 40 individuos, pertenecientes al Municipio de Betulia (Sucre) de características sociodemográficas similares, con la finalidad de probar en el campo los instrumentos de evaluación y a su vez entrenar a los investigadores y verificar el manejo del instrumento. Los resultados permitieron realizar ajustes y modificaciones en algunos criterios y reestructurar los instrumentos. De corte cuantitativo se utilizó una encuesta o cuestionario, con el fin de conocer las características sociodemográficas de la pobla- 


\begin{tabular}{|l|c|c|c|}
\hline \multicolumn{1}{|c|}{ Municipio } & Poblaciones & Porcentajes & Muestras \\
\hline San Onofre & 1.020 & $2 \%$ & 6 \\
\hline Tolú & 1.350 & $2 \%$ & 8 \\
\hline Colosó & 194 & $0 \%$ & 1 \\
\hline Sincelejo & 34.214 & $56 \%$ & 212 \\
\hline Toluviejo & 5482 & $9 \%$ & 34 \\
\hline Sampués & 19.384 & $31 \%$ & 120 \\
\hline Totales & 61.644 & $100 \%$ & 381 \\
\hline
\end{tabular}

manera sistematizada mediante el empleo de códigos que obedecían a las subcategorías antes mencionadas y a sus respectivos criterios de análisis, ejemplo: FOR-FON (corresponde a la subcategoría de forma y el criterio de fonología), CON-COM (subcategoría de contenido y procesos de comprensión) y USO-CONV (subcategoría uso y criterio de conversación), entre otros.

Teniendo en cuenta las características del proyecto, su temporalidad y lógica, se delimitaron las siguientes fases: a) Demarcación del campo, donde se eligió la comunidad del resguardo de San Andrés de Sotavento, teniendo en cuenta los intereses investigativos, los supuestos y principios epistemológicos, los propósitos que se persiguieron, el tiempo, las actividades, los recursos materiales y las técnicas que se utilizaron y elaboraron. Así mismo, se solicitó la autorización ante las autoridades indígenas, y seguido se efectuó una prueba piloto para la validación de los instrumentos. b) Trabajo de campo, en la cual se ingresó al escenario investigado, aplicando los instrumentos destinados para la recolección de la información. Previo a esto, se solicitó el consentimiento informado a los sujetos de estudio o persona responsable, siguiendo los lineamientos éticos dispuestos por el comité de ética de la División de Investigación de la Universidad de Sucre (DIUS). c) Análisis cualitativo y cuantitativo, discusión y elaboración del informe final, en esta etapa se analizaron los resultados generados en el trabajo de campo, y posteriormente, se elaboró el informe final que fue socializado con la comunidad y otras instancias.

\section{RESULTADOS}

\section{Características Sociodemográficas de la Población Zenú}

Los resultados dejan ver que en cuanto a género, la población se encuentra equitativamente distribuida, pues aproximadamente la mitad pertenece al género masculino y la otra al femenino, siendo ligeramente superado en número este último; cuyas edades se encuentran en su mayor porcentaje entre 19 años en adelante $(52.7 \%)$ y en menor proporción entre los 13-18 años (12.9\%). El nivel educativo tiene un comportamiento heterogéneo según los municipios estudiados, encontrando que en Sampués, San Onofre, Sincelejo y Colosó la mayoría de los habitantes alcanzan la primaria incompleta (37.1\%), seguido de aquellos que nunca se han alfabetizado (21.7\%); por el contrario, en los municipios de Toluviejo el mayor porcentaje alcanza bachillerato incompleto (41\%), mientras que en Tolú un número significativo no ha estudiado (33.9\%).

En esta comunidad predominan las familias integradas por padres e hijos (57.4\%), conformadas en su mayoría entre 4-6 miembros (55.2\%), y en menor instancia por los hogares con más de 8 personas (7.1\%). Encontrándose que el jefe del hogar es corrientemente el hombre quien labora como agricultor $(30.1 \%)$. Las familias por lo general poseen residencia propia, conviven en moradas tipo casa, con características tradicionales del caribe colombiano, construidas con techo de palma, horcones de madera, paredes con guadua, caña y bahareque. Las condiciones de la vivienda, en cuanto a servicios públicos son precarias puesto que solo cuentan con energía eléctrica y acueducto, careciendo de los demás servicios públicos. 
Para efectos del análisis, las particularidades fonético - fonológicas se identificaron atendiendo al concepto de "procesos fonológicos”, descritos por Acosta et al. (2002) como restricciones del habla que aparecen durante el desarrollo evolutivo y suelen suprimirse paulatinamente. Sin embargo, teniendo en cuenta que la población objeto de estudio supera la etapa evolutiva del lenguaje, no se mirarán como alteraciones en este nivel sino como "errores socioculturales”, que según los mismos autores son limitaciones del habla debidas a la influencia del contexto personal de los individuos como por ejemplo /pa/ por/para/, /pos/ por/pues/, /jalar/ por/halar/, entre otros. Así mismo, Herrera y Butragueño (2008) los explican por la influencia que los contextos tiene sobre los cambios fónicos o formas de expresión los cuales se materializan en tendencias de simplificación y manifiestan continuidad a lo largo del tiempo.

En este orden de ideas, los procesos fonológicos según Acosta et al. (2000) se clasifican en aquellos relativos a la estructura silábica, a la asimilación y a la sustitución. Los procesos de estructuración silábica tienden a reducir la silaba al esquema consonante vocal (CV) o a simplificar el número total de silabas que componen una palabra. Los procesos de asimilación, son aquellos en los que se sustituye un segmento por otro que se encuentra en la misma palabra y los procesos de sustitución, como su nombre lo indica, son los procesos por los que se sustituyen clases enteras de sonidos sin hacer referencia a los sonidos más próximos.

De acuerdo a los resultados la mayoría de la población utiliza procesos de asimilación, dichos procesos son generalmente regresivos, porque el elemento influyente se encuentra después del influido $y$, adyacentes o contiguos porque están próximos, siendo evidentes asimilaciones de los sonidos $/ \mathrm{r} / \mathrm{y} / \mathrm{l} /$ en posición inversa, igualmen-

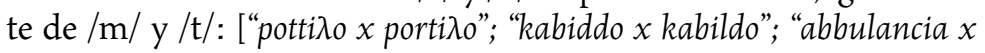
ambulancia"; "kokkobea $x$ korkobea"; "abakka $x$ abarca"; FOR- FON].

Es así como, en sus conversaciones son usuales expresiones que tipifican los procesos de asimilación: velar ["aggunas" x algunas FORFON]; alveolar ["patilla" x partirla; FOR-FON]; nasal ["baxamme" x baxarme; FOR- FON] y bilabial ["kueppo" x cuerpo; "FOR- FON].

Un fenómeno muy frecuente, dentro de los procesos sustitutorios fue la aspiración de trabante, por el cual se aspira un sonido que traba la sílaba cuya estructura corresponde a CVC, específicamente cuando se trata del fonema/s/, ["nosotroh" x nosotros; "unah" x unas, "ehto" x esto; FOR-FON]. Este caso sucede en distintas posiciones de la palabra; aunque, se presentó con mayor frecuencia en sílaba final.

De igual forma, se hallaron procesos de: sustitución de vocales entre sí [“dispues" x después; FOR- FON]; frontalización ["tru” x cruz FOR-FON]; neutralización o sustitución de vocales por /a/: ["axuala" ${ }^{1}$ x ojalá; FOR- FON]; sustitución de vibrantes por laterales:

1. En el vocablo además del proceso señalado de sustitución de vocal, se aprecia una adición de la vocal /u/ en la segunda sílaba, es decir, expresan "ajualá" por "ojalá"; por consiguiente, la transcripción del diptongo es de acuerdo a la expresión encontrada: ["axuala” x ojalá]. ["sordado" x soldado; FON PROAL]; lateralización de vibrantes: [ "salten" x sartén FOR- FON]; lateralización de oclusivas ["fulbolista" x futbolista; FON PROAL] sustitución de oclusivas por laterales [

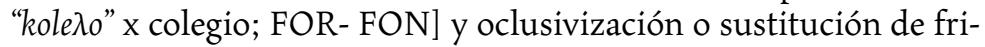
cativas por oclusivas sordas ["relot" x reloj FON PROAL].

Así mismo, se pudo determinar la presencia de procesos en los que se tiende a reducir la silaba al esquema consonante vocal (CV) o vocal (V), es decir, procesos de estructuración silábica, que se materializan con omisiones o reducciones. Ejemplo de lo anterior, es que la población se caracteriza por realizar procesos de omisión de la consonante final /r/, especialmente en la silabas del tipo CVC, representando el caso más característico de la expresión Zenú y evidenciándose especialmente en verbos en infinitivo, por ejemplo:

["rekuperá" x recuperar; "trensá" x trensar; "desî́ x decir, FOR- FON].

Hubo además procesos de omisión de la consonante /d/ en posición inicial de silaba final de la palabra, presentando alta incidencia en sustantivos y participios verbales, tales como: ["too" $\mathrm{x}$ todo; "aguantao" x aguantado; "soldao" x soldado; "kandao" x candado FOR-FON]; igualmente, omisión de /d/ en posición final de silabas con estructura CVC al final de la palabra [ "berdá" x verdad FOR- FON]; la omisión de /d/ se apreció incluso en otras posiciones ["onde" $\mathrm{x}$ donde; "e" $\mathrm{x}$ de FOR-FON]; también se precisaron reducciones de silabas finales con estructura de CVC por CV ["reló" $\mathrm{x}$ reloj; FOR- FON]; omisión de consonante inicial en sílabas átonas ["to'o" x todo; "pa'a" x para FOR-FON] y epéntesis o inserción de sonidos dentro de la palabra ["asienta" x sienta; "abaxito" x bajito FOR- FON].

En cuanto al carácter morfosintáctico del lenguaje la población emite construcciones bimembres y unimembres; es decir, emplean oraciones simples de diferente composición: ["yo nací aquî"; "soy ama de casa"; "es una pulsera" FOR MOR] y oraciones complejas, preferentemente yuxtapuestas y coordinadas, pero escasamente subordinadas ["yo únicamente del campo, trabajá, sembrá yuca, sembrá mai, raspá, pilá, lavá, cociná"; "pensé que no iba a suceder más y dejé eso quieto"; "Ahora llegó el pescado y se devolvió"; "esta agua que está aquí se está derramando" FOR MOR]. Según la actitud del hablante, todos hacen construcciones enunciativas (afirmativas y negativas), empleando vocablos "sí" y "no" ["si tengo"; "no alcanza" FOR MOR]. Contrariamente, no es usual la elocución de oraciones interrogativas, exclamativas, y desiderativas aunque suelen presentarse en mínima proporción [“icómo reaccionaría?; ";Ay Dios mío!"; "deseo seguirme capacitando” FOR MOR]. Mientras que, fueron nulas expresiones dubitativas, atributivas e imperativas.

Sobre los elementos gramaticales, la población hace uso de palabras de primera clase, secundarias y de relación; sin embargo, en ocasiones se manifiestan accidentes relacionados con el orden gramatical, presentándose transposiciones, sustitución u omisión de elementos oracionales, que varían la inteligibilidad del discurso: ["uno por acá el método de uno es la escubilla"; "yo ello llamo Luis"; "porque que vamos a decir que decimos que tenemos todo" FOR MOR]. Igualmente establecen relaciones adecuadas entre los morfemas flexivos que indican diferencias de género, número y persona en los sustantivos, 
adjetivos, verbos y pronombres que conforman el discurso, permitiendo la concordancia y coherencia de lo expresado. Sin embargo, se observan producciones inconcordantes de número, más que de género, sobre todo cuando el enunciado es extenso o denota desconocimiento del tema. ["la cabro"; "unos perro"; FOR MOR].

La conjugación verbal característica de la población Zenú es la compuesta por convenciones verbales no personales en infinitivo y gerundio: ["caer", "golpear"; "gritando"; "esperando" FOR- MOR]; asimismo, conjugaciones en modo indicativo más que subjuntivo, destacándose el uso mayoritario de tiempos presentes y pasados (pretérito); moderado de tiempos copretérito y pospretérito y casi nulo en futuro ["me tuvo", "yo nacî"; "está lavando", "me levanto", "yo veo", "él vivió"; "me siento", "me fui" "yo tengo", FOR-MOR]. La población escasamente hace uso de construcciones en tiempos verbales compuestos y producciones de modo indicativo, más que subjuntivo: ["yo ha dicho"; "he visto", "he hecho";...FOR-MOR]. Del mismo modo, se aprecian particularidades en las conjugaciones verbales, que se encuentran arraigados en la cultura costeña: "haldría" por "haría", "semos" por "somos"; "aparecen" por "parecen"; "se ñama" por "se Ilama"; "dicir" por "decir"; "lo ha hecho" por "lo he hecho"; "haiga por haya"; FOR-MOR].

\section{Caracterización de la dimensión de Contenido}

En cuanto a las particularidades en los procesos de comprensión, la colectividad se caracteriza porque hacen reconocimiento por señalamiento y nominación de imágenes concretas, aunque algunas veces son sustituidas por vocablos relacionados con el referente y cuando lo desconocen coligan su concepto a través de la asociación con otro conexo de diferente significación, pero con semejante forma o característica física ["carro por ambulancia"; "rula" por "machete"; "mula" por "caballo"; "hicotea" por "tortuga"; "campana" por "lupa"; CON-COMP]. Por otra parte, emplean vocablos contextualizados que dan cuenta de la riqueza semántica propia de su cultura, relacionándolos comúnmente con el oficio de la artesanía, la naturaleza y su identidad étnica (Ver tabla 2).

Los Zenúes demostraron habilidades para clasificar vocablos estableciendo relaciones semánticas coherentes y reuniendo elementos conforme a su entorno sociocultural; sin embargo, se evidenció un vocabulario léxico receptivo limitado y básico, atendiendo a lo conceptuado por Herrera, Gutiérrez y Rodríguez (2008), caracterizado por el uso de términos vagos o genéricos en lugar de términos específicos, ejemplo [“dame la cosa que está ahî"]. Por otro lado, la mitad de la población presentó poca correspondencia para establecer conexiones semánticas entre palabras con significados iguales y contrarios, denotándose mayoritariamente en sinónimos [Sinónimos: "bonito-cargado"; "sembrar- ordeñar"; "hacer-destruir"; Antónimos: "triste-no se"; "lejos-alejado"; "hablar-narrar"; CON-PRO]. Igualmente, se particulariza por hacer compensaciones a la hora de relacionar conceptos, como por ejemplo: "asear-vestir" por "asear-limpiar"; "bonita-buena" por "bonita-hermosa; como también, emplean otras palabras incluidas en la familia semántica para hacer la correspondencia solicitada, ejemplo: "triste-tristeza; "mucho-muchísimo".

En el plano de las cualidades de los procesos de producción, se encontró que la mayor parte de los usuarios hacen mención de los

\begin{tabular}{|c|c|c|}
\hline Vocablo & Transcripción Fonética & Definición \\
\hline Pilar & /pilal & Moler en pilón \\
\hline Hombe & lombe/ & $\begin{array}{l}\text { Abreviatura de hombre incluida en } \\
\text { el costeñol }\end{array}$ \\
\hline $\begin{array}{l}\text { Sombrero } \\
\text { vueltiao }\end{array}$ & /sombrero bueltiao/ & $\begin{array}{l}\text { Artesanía elaborada en caña fle- } \\
\text { cha y considerada obra de arte y } \\
\text { legado Zenú al patrimonio nacio- } \\
\text { nal. }\end{array}$ \\
\hline Me salí & Ime sali/ & $\begin{array}{l}\text { Afirmación empleada cuando } \\
\text { la mujer se compromete con un } \\
\text { hombre y deja la casa de sus pa- } \\
\text { dres }\end{array}$ \\
\hline Trenzar & /trens'a/ & $\begin{array}{l}\text { Arte de armar en trenzas la palma } \\
\text { de caña de flecha }\end{array}$ \\
\hline $\begin{array}{l}\text { Abarca } \\
\text { tres puntá }\end{array}$ & labbacca tre'e punt'al & $\begin{array}{l}\text { Tipo de calzado de estilo sandalia } \\
\text { elaborado con el cuero de la vaca }\end{array}$ \\
\hline Zoco, rula & /soko, rula/ & $\begin{array}{l}\text { Variantes de machete usual en el } \\
\text { caribe colombiano }\end{array}$ \\
\hline Hicotea & /ikotea/ & $\begin{array}{l}\text { Tortuga que habita en la zona ce- } \\
\text { nagosa norte de Colombia }\end{array}$ \\
\hline Iraca & lirakal & $\begin{array}{l}\text { Palma cuyo nombre científico es } \\
\text { Carludovica palmata, que nace en } \\
\text { zonas templadas y eje Cafetero } \\
\text { del país, que sirve para la elabo- } \\
\text { ración de artesanías. }\end{array}$ \\
\hline
\end{tabular}

Fuente: Consolidado de muestras de lenguaje de la etnia Zenú (caracterización del lenguaje oral de la población Indígena Zenú-Sucre de 2012.

Tabla 2. Vocablos contextualizados empleados por la población Zenú.

elementos que componen una lámina y establecen relaciones coherentes entre ellos; sin embargo, una parte menos significativa de la población, confunden personajes, no establecen relación análoga entre ellos, ni abstraen su significado, pese a recibir un estímulo verbal activo. Asimismo, los razonamientos que utilizan tienden a ser inconsecuentes en la medida en que no establecen relaciones causales fundamentales, sino arbitrarias y secundarias; por ejemplo, ante el interrogante de ipor qué la señora deja derramar el agua?, expusieron juicios como: "será porque no le sirve la pluma"; "porque tiene la pluma abierta"; entre otras, antes que su razón principal: "debido a la distracción de la señora"; no obstante, toda la población hace predicciones acertadas y discusiones asociadas relacionadas con su contexto natural.

En lo concerniente a descripción, la mayoría de la población Zenú representa verbalmente objetos según sus atributos físicos y materiales, más que en sus cualidades de uso, elaboración y significación cultural: "está hecho de palma, de color rapé y blanco"; "es redondo, tiene hilo pegado alrededor, ... CON-PRO]; aun así, algunos pobladores de la etnia Zenú hacen descripciones expansivas de los objetos, resaltando de ellos sus cualidades afectivas como artesanos, su valor y trascendencia cultural para la sociedad: ["esto lo hacen aquí en Sampués, son cosas artesanales, también las hacen en Lorica, Tuchín, San Andrés y ya las conoce todo el país"... CON-PRO].

Por otra parte, en la descripción de los tipos de significados, donde se hizo alusión inicialmente al significado referencial o léxico; se encontró que la totalidad de los sujetos valorados durante su dialogo 
emplearon diversos elementos gramaticales con significado nocional y funcional, conjugando tanto en construcciones simples, como complejas. A nivel del significado semántico, se pudo encontrar que la población se caracteriza por elaborar conceptos concretos partiendo de rasgos de funcionalidad y percepción, pero no los enuncian a través de campos semánticos específicos: ["moler es triturar"; "una tualla es pa' secarse"; "la luna es blanca";... CON-SIG]; ahora bien, es usual que hagan sobreextensiones para definir cuando no precisan los elementos para organizar una producción oral, por ejemplo: ["tabla con que se hacen las puertas"; "luna la que sale en las noches y uno la ve"; "mariposa es un animal"; "'toalla es tela";... CON-SIG]. Todavía más, para elaborar conceptos abstractos, se caracterizan por dar apreciaciones afectivo-emocionales según experiencias vividas o deseadas: [Definición de felicidad: "es de pronto cuando a usted le dan una buena noticia"; Definición de sinceridad: "es cuando uno está hablando con una persona, hay veces que uno tiene problemas..."; Definición de amor: es tenerle amor a sus hijos, al marido"...CON-SIG] .

Además se pudo encontrar con base a los significados figurativos, que la mitad de la población Zenú establece relaciones semánticas a partir de un referente poco usual y tienen destrezas para interpretar el lenguaje en expresiones metafóricas; no obstante, la otra parte de la población se particulariza por hacer interpretaciones literales y equívocas de contenidos alegóricos: [concepto de cabeza dura: "que es duro para todo"; concepto de estar en la luna: "que es necia"; concepto de ángel: "que hace caso, los mandados" ; ...CON-SIG]. A través de las muestras se pudo percibir que la población indígena en su totalidad mantiene una cohesión discursiva caracterizada por concordancia de los elementos gramaticales; percibiendo en el discurso la coherencia global y local, siendo la coherencia secuencial medianamente visible.

\section{Caracterización de la dimensión de Uso del lenguaje}

La población Zenú se caracteriza en su mayoría por no iniciar interacciones en una conversación, sin embargo logran mantener, esperar y ceder tiempos; en cuanto al dominio y elección del tema la mayoría optó por exponer tópicos personales sobre sucesos familiares vividos, labores diarias y anécdotas. En lo relacionado al compromiso conversacional, es decir, el grado de implicación en la conversación según el tipo de respuesta y de la cantidad de información proporcionada (Acosta et al, 2002), se pudo determinar que la población indígena Zenú (sobre todo niños) se caracteriza por dar respuestas básicas y cortas que hacen necesario para el interlocutor emplear reguladores o continuadores de la conversación. ["I: ¿cómo estás Melissa?" E: "bien"; "I: cuénteme za qué se dedica usted?" E: "a na"; "I: ¿Cuando tú eras niño también eras así de necio? E: "no"... USO-CONV]. En su totalidad emplean elementos deícticos de persona, ejemplo: "yo únicamente del campo... y atendé a mis hijitos, porque después que vengo del monte me toca hacer todo eso". Así mismo usan elementos deícticos de lugar "yo veo que estos aquí, aquí los veo que están como... pilando, aquí está una niña, un niño y acá está un mujé está teniendo un plato ahí en la cocina". De igual forma, usan deixis de tiempo, ejemplo: "enseguida como no hay gas, después viene ajá la lavandina de plato, después otra vez el almuerzo, hoy hice bollo de plátano".

Hacen uso de la expresión acción con sus actividades cotidianas como cargar objetos, lanzarlos o moverlos, que involucran mo- vimientos de brazos, manos y posturas específicas, también de la expresión gestual con gestos naturales como: movimientos de los ojos, sonrisas, fruncir el ceño, entre otros, los que utilizan a la hora de describir o hablar; y en la expresión verbal usan sonidos, sílabas y polisílabas, pero como rasgo distintivo hablan rápido, cambian la entonación de palabras como: "maíz por mái"; "ahí por áhi"; "peladito $x$ peláito", entre otras y el volumen de su voz es normal aunque en niños y mujeres se denota un poco más bajo.

Durante su comunicación es usual el empleo de gestos espontáneos que son comunes en diferentes culturas y regularmente se utilizan al saludar, despedirse, asentir y negar, pero a la hora de usar gestos deícticos incluyen en su cotidianidad ademanes de señalar, ya sea a ellos o a los objetos que referencian en su contexto lingüístico (Ver Figuras 1, 2 y 3). Sin embargo existen algunas personas muy expresivas que se empoderan de la deixis a través del gesto para darle mayor significación a su acto lingüístico (Ver Figuras 4, 5, 6 y 7).

\section{Al hablar de sí mismo o "Yo"}

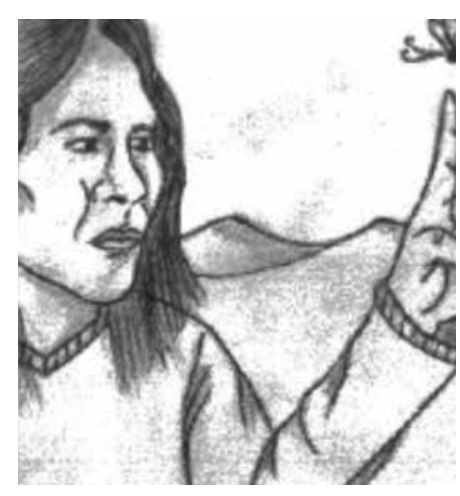

Figura 3.

Al hablar de otra persona (él o ella)

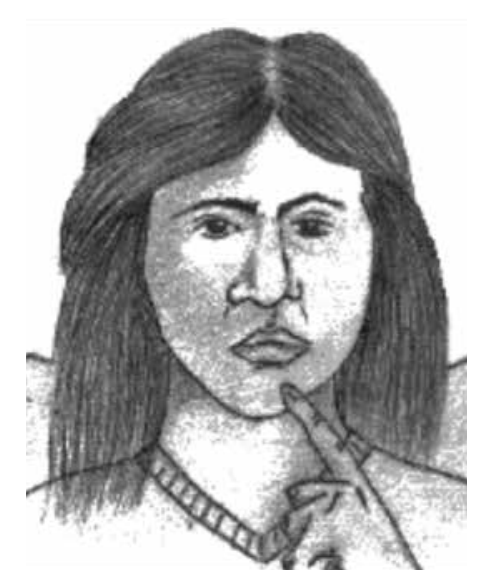

Figura 2.

Al hablar de otro (objeto)

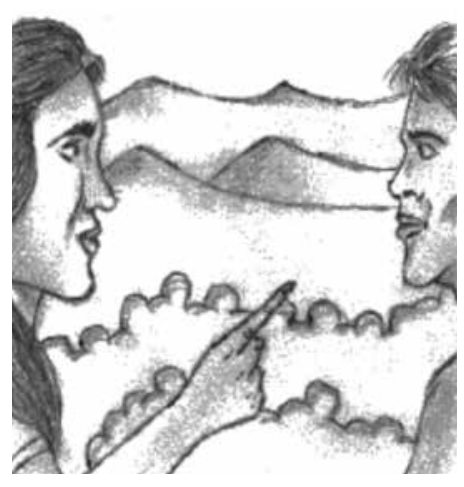




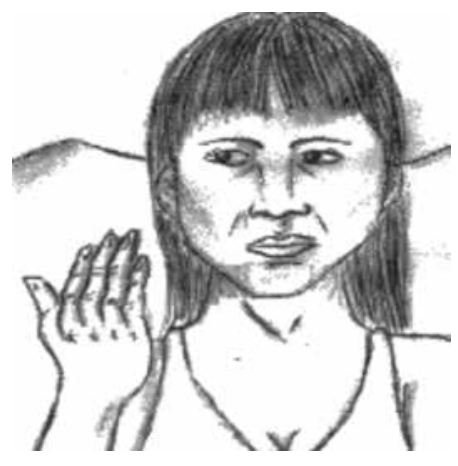

Figura 4.

Movimientos de las manos hacia atrás que denotan el pasado.

Figura 5. movimiento de balanceo de la mano expresando "más o menos"

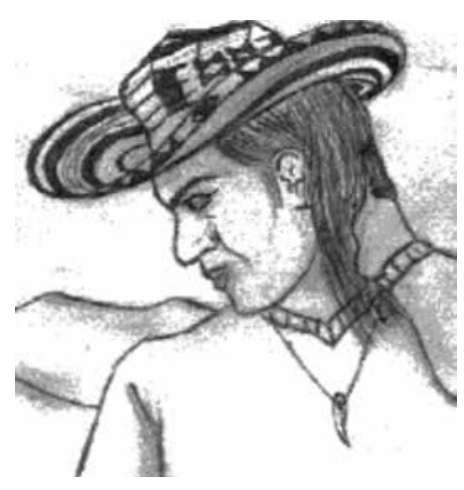

Figura 7 .

Colocarse la mano en la barbilla o el dedo a la boca expresando estado pensativo. pesan estas interrupciones con el uso frecuente de muletillas tales como: "ehhh, uhhh, ahhh, esteee, mmmm, ajá, siii, ujú, ummm", entre otras, y en ocasiones se evidencian silencios prolongados. Es importante anotar que las interrupciones pueden deberse a fallos de significado o de frecuencia que produce falta de continuidad en las emisiones, por consiguiente no pueden constituirse como descortesías o fracasos comunicativos.

\section{DISCUSIÓN}

El lenguaje de la población indígena Zenú, desde la dimensión de forma, se caracteriza por presentar persistencia de "errores socioculturales" que para efectos de ésta investigación fueron descritos como procesos fonológicos según los lineamientos de Acosta et al. (2002) quienes los definen como restricciones del habla que aparecen durante el desarrollo evolutivo y que suelen suprimirse paulatinamente, pero que pueden permanecer luego de superar la etapa evolutiva del lenguaje, por influencia del contexto.

Las asimilaciones referenciadas se presentan en fonemas contiguos pues tal es la influencia de uno sobre el otro que crea una forma de facilitación en la producción de las sílabas, principalmente aquellas de estructura CVC, dando lugar a un fenómeno de sustitución, más no de omisión, como comúnmente se cree. Se destaca que estas particularidades son las que caracterizan la forma del lenguaje oral de la población y le otorgan lo que regionalmente llaman "el acento golpeado", vinculándose con lo expuesto por Paternina (2002) quien acopia vocablos de la población Zenú cordobesa que contienen procesos de simplificación símiles con los encontrados en sus homólogos de Sucre.
Figura 6.

movimiento de frotarse la mano por la zona cervical expresando incomodidad

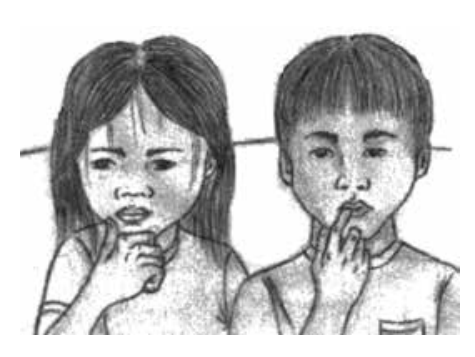

Adicionalmente, la mayoría de la población se caracteriza, por hacer contacto corporal durante el saludo con el gesto espontáneo de dar la mano, mantienen una distancia prudente al entablar la conversación, aunque el contacto visual no es habitual en ellos; lo que se tradujo en miradas esquivas hacia direcciones y polos opuestos (mirada al piso o al techo) durante el acto del habla.

En cuanto a los rasgos de la fluidez del discurso la mayoría de la población no emplea autocorrecciones pero es habitual la presencia de interrupciones dentro de las conversaciones, creando lapsos o espacios entre los intercambios comunicativos, sin embargo, so-
Se identificaron además procesos de sustitución que dan cuenta de la riqueza cultural de la expresiones en la comunidad Zenú, coincidiendo con lo encontrado por Buitrago et al. (2007), Sánchez et al. (2002) y Albán et al. (2007) en la producción oral de otras poblaciones indígenas del país; y ratificando lo citado por Turbay y Jaramillo (2000), sobre la conservación de la " $h$ " aspirada del español antiguo.

Los procesos de estructura silábica presentes en la población, se explican desde lo descrito por Paternina (2002), quien indica que los indígenas de este resguardo en Córdoba, expresan vocablos que contienen omisiones o reducciones fonémicas. Sin embargo, Aleza y Enguita (2010) afirma que en Colombia se destaca la tendencia de la aspiración, que puede dar lugar a fenómenos como las asimilaciones de las consonantes siguientes con la pérdida de la "s" y de la "r" en las zonas costeras que se extiende a numerosos países. Además, neutralizaciones entre la vibrante y la lateral "l", siendo para las regiones costeras de Colombia más común la neutralización en favor de la lateral, al igual que los fenómenos de eliminación de consonantes siguientes (Cat-tagena), de esto puede inferirse que los procesos fonológicos de la región costera probablemente pudieron indígenas. En concordancia, Trillos (2001) afirma que existen rasgos que caracterizan el español suramericano determinando nueve zonas lingüísticas; ubicándose en la primera el caribe colombiano cuyo distintivo es la aspiración o pérdida de la /s/, articulación glotal de $/ \mathrm{j} / \mathrm{y}$ presencia de tuteo. haber sido influenciados por los legados culturales de los ancestros 
En cuanto a las cualidades morfosintácticas, la carencia del uso de oraciones dubitativas, atributivas e imperativas, puede relacionarse con el contexto lingüístico elicitado; dado que, la comodidad que puede sentir la persona para expresar sentimientos o ideas mediante frases con inflexiones, dependen de la naturalidad del contexto lingüístico y ello explica el por qué la población puede hacer uso de ellas al hablar con sus hijos u otros en entornos diferentes. Así mismo, la presencia de accidentes gramaticales de orden y concordancia, resulta correspondiente con lo encontrado por Buenrostro (1998) y Gallego y Arboleda (2004) en poblaciones étnicas; dado que, fueron evidentes las alteraciones en el orden gramatical y carencia de elementos sintácticos como preposiciones, artículos y verbos; sin embargo, en este estudio, éstas no fueron determinantes para la coherencia discursiva y probablemente se debieron a la poca contextualización con los temas lo que disminuye la seguridad, cohesión y concordancia en el contexto verbal.

Se puede inferir además, que la forma como cada comunidad concibe su cosmovisión, le permite percibir su diario vivir y el futuro; así, la escasez de verbos en conjugación futura de la población, se debe posiblemente a que su prioridad es la provisión de las necesidades en el "día a día" y no existe un interés apremiante por las futuras o del mañana. Por último, la presencia de particularidades de conjugación en la población, dan cuenta de fenómenos populares, probablemente debidos al mosaico étnico-lingüístico-cultural, que luego de la dominación española, se ha convertido en un castellano impreso de creatividad lingüística que singulariza a los indígenas Zenúes. Así mismo, coincide con Paternina (2002) quien afirma que esta etnia se caracteriza por poseer abundancia cultural de los vocablos verbales autóctonos del resguardo San Andrés de Sotavento como: "dir" por "ir", "jiede" por "hiede", "juye" por "huye", "juntá" por "untar", entre otras.

Ahora bien, analizando los resultados de la dimensión de contenido la presencia de un vocabulario léxico limitado no afecta la coherencia con el referente semántico de la conversación ni el éxito de la misma; lo que se correlaciona con los postulados de Bernstein y Bruner, (citados por Gallardo y Gallego, 200o) quienes ligan el desarrollo de los significados a partir de las clases sociales y la cultura, respectivamente, fundamentos importantes en este contexto dado que, sociodemográficamente ya se expusieron las privaciones de la población al respecto. Para Bernstein el individuo desarrolla un repertorio semántico elaborado si su contexto se lo provee. Bruner, afirma que las familias deprivadas culturalmente van perfilando un tipo especial de código lingüístico, a veces limitante para el medio escolar en la construcción de conocimientos a través de la codificación científica. Lo anterior no limita la variedad cultural del vocabulario de la población indígena Zenú, tal como se explicitó en la tabla 2, propia de su experiencia y práctica sociocultural.

Se determinó además la poca correspondencia para establecer conexiones semánticas entre palabras, siendo coherente con lo expuesto por Vygotsky (1995) quien expone que la aprehensión de las diferencias precede a la de las semejanzas, porque la toma de conciencia de la similitud requiere una estructura de generalización y conceptualización más avanzada que la exigida por la de diferencia.
Por otro lado, las características para analizar la significación guardan relación con lo expresado por Oleron (1985) quien explica que la definición de significación, no solo se establece en un vínculo entre palabras y referentes, sino en su dimensión fundamental de comunicación, que implica no solo que se hable de algo, sino también que, en sus palabras hablante y oyente se tengan en cuenta y se entiendan, el uno al otro. El uso de conceptos concretos sin utilizar campos semánticos específicos, guarda relación con lo expuesto por Luria (1985) quien afirma que una conceptualización no es una asociación simple y unívoca entre la señal sonora convencional y la representación directa, dado que ella posee una multitud de significados potenciales que se encuentran inmersos tanto en su estructura morfológica, como en su uso práctico en la vida cotidiana, por ejemplo: la palabra "carbón", para un pintor significará "un utensilio para pintar"; para la ama de casa será "un medio con el que se enciende la estufa of fogón". Incluso, la población en general hace sobreextensiones o utilizan la misma palabra al conceptualizar conceptos abstractos, para subsanar la falta de argumentos, ejemplo: para definir felicidad expresaron: "Es estar feliz"; para definir sinceridad se refirieron: "sinceridad, uno tiene que ser sincero con otro"; para definir bonito utilizaron: "bonito es aquello que es bonito, lindo"; entre otros.

De igual forma, se puede inferir que el predominio figurativo concreto o literal se debe al fenómeno expresado por Luria (1985) que consiste en que el individuo separa y cuenta palabras que designan objetos concretos, pero omite las que tienen un significado auxiliar e inconcreto, lo que de alguna manera, lo lleva a hacer interpretaciones totalmente literales. En el caso de la etnia Zenú no se trata de anormalidades de su comprensión, sino particularidades de su forma de elaborar los conceptos; los significados figurados, al igual que todos los aspectos semánticos, se ven influenciados por el contexto sociocultural en la medida en que la tradición oral de los ancestros deriva a conceptualizaciones místicas de algunos de ellos. La población indígena mantiene una cohesión discursiva lo que puede deberse, según Acosta y Moreno (1999), no es necesario que la forma del discurso sea adecuada para transmitir los significados que se quieren expresar pues el diálogo no es una mera secuencia de oraciones, por el contrario se constituye en un todo articulado.

Para el análisis del uso del lenguaje se tuvo en cuenta que para llevar en buenos términos una conversación no sólo se necesita tener claridad conceptual del tema, sino tener una actitud dispuesta, un desarrollo oportuno y cortés, que demanda de los participantes habilidades y destrezas. Para el caso de la población analizada se concluye, en cuanto a las destrezas conversacionales, que al tratarse de una muestra del lenguaje para fines investigativos, la naturalidad del clima conversacional se afecta, e imponer un ligero condicionamiento hizo que los participantes pudieran estar muy pendientes de su actividad verbal, de la grabación y de los espectadores que la presenciaran; esto se correlaciona con lo expuesto por Hofstadt (2005), quien afirma que "iniciar una conversación cuando no conocemos a nuestro interlocutor o cuando nuestro conocimiento es mínimo supone una tarea que prácticamente a todo el mundo le resulta extremamente complicada".(p. 176)

Otra particularidad son las interrupciones representadas en el uso de muletillas y silencios en la conversación, que pueden ana- 
lizarse como rupturas conversacionales; dado que, se producen a mitad de una unidad construccional de turno, lo que supone un rompimiento considerable de la corriente de habla, que a su vez puede deberse a la falta de naturalidad en la conversación y a los rasgos de personalidad de la población étnica. Foley (2004), desde su investigación sobre la comunicación de los indígenas Mesquakis, puede servir de correlación, para el análisis del uso de los silencios, en la medida que el autor describe la actitud del silencio como una característica que identifica a la comunidad étnica estudiada, lo que los lleva a identificarse como personas silenciosas, nobles y dignas, contrario con el lenguaje estrepitoso, grosero y desprevenido de los jóvenes blancos. Para Haverkate (1997) el silencio puede interpretarse como una función metalingüística con otra intención que puede ser una señal de la cortesía ritual manifestada por personas de categoría social inferior, por consiguiente en la antigüedad no era extraño, que en familias jerárquicamente organizadas obligaban a sus hijos a callar hasta que ellos mismos les confirieran el permiso de hablar, convirtiéndose esto en una etiqueta que necesariamente regulaba la dinámica conversacional; situación que en la actualidad, en diferentes esferas sociales aún son comunes y de las cuales las comunidades étnicas no están exentas.

Los indígenas Zenues prefieren temas triviales y cotidianos a la hora de proponerlos, esto se relaciona con lo afirmado por Siebold (2008), quien denota que optar por un tópico encierra un gran potencial de conflicto, ya que elegirlos incorrectamente puede dificultar la comunicación, así mismo este autor expone que de acuerdo a las culturas existen tópicos que son prohibiciones y puede esto interferir el proceso comunicativo si se desconoce. En cuanto al compromiso conversacional es evidente la producción contingente simple que se caracteriza porque el sujeto proporciona información mínima para que no se rompa la conversación y que puede deberse a particularidades socioculturales en el comportamiento conversacional. (Acosta et al. 2002)

Se pudo inferir que todas las características fónicas como volumen, tono, cadencia al hablar son indicadores de la procedencia de la persona, del medio ambiente en que se cría, de su carácter, de su estado psíquico, etc., sin embargo, no existen evidencias escritas sobre las descripción de los rasgos gestuales de los indígenas Zenues. Por consiguiente, las particularidades de la expresión verbal son las que representan a los procedentes de la región Caribe de nuestra nación.

La población en general, no se autocorrige debido a su vocabulario y sintaxis limitada, las personas no se encuentren familiarizadas con ciertas combinaciones fonéticas, semánticas o sintácticas poco habituales, por consiguiente si las expresan mal, no resulta significativo a la hora de autocorregirse (Oleron, 1985).

\section{CONCLUSIONES}

La población indígena Zenú de Sucre se caracteriza a nivel educativo por un comportamiento heterogéneo entre municipios, pre- valeciendo los niveles de primaria incompleta y analfabetización; las familias en su mayoría conforman hogares nucleares y nutridos, predominando la unión libre; el jefe del hogar es el hombre quien generalmente trabaja informalmente, poseen viviendas propias con techo de palma y paredes de bahareque.

La población indígena crea estrategias para suplir contrastes fonológicos atribuibles al contexto sociocultural, que se materializan en procesos de asimilación en fonemas contiguos, como en sonidos alveolares $/ \mathrm{r} /$ y $/ \mathrm{l} /$ en posición inversa y otros. En el discurso se presentan sustituciones de clases enteras de sonidos, como la aspiración del fonema /s/ y omisión de /r/ final en la silabas del tipo CVC y de /d/ inicial en sílaba final. Se evidencia el empleo de oraciones simples y complejas de tipo yuxtapuesta y coordinada, siendo escasas las subordinadas; según la actitud del hablante, construyen oraciones de todo tipo excepto dubitativas, atributivas e imperativas, guardando concordancia gramatical en los aspectos morfológicos que las contienen. La conjugación verbal se da mayormente en modo indicativo, utilizando las formas simples conjugadas en presente, pretérito y copretérito, pero escasamente en futuro, conservando algunas particularidades de conjugación propias de fenómenos populares debidas al mosaico étnico-lingüístico-cultural que luego de la dominación española se ha convertido en un español impreso de creatividad lingüística que singulariza a los indígenas Zenúes.

A nivel de la dimensión de contenido del lenguaje los indígenas se caracterizan por emplear vocablos acordes a su entorno cultural, dejando entrever un vocabulario receptivo básico, lo cual puede estar relacionado con la poca correspondencia en el establecimiento de conexiones semánticas. Al expresar conceptos concretos utilizan rasgos semánticos de funcionalidad y percepción, mientras que, al definir palabras abstractas utilizan apreciaciones afectivo- emocionales y sobreextensiones. A pesar de estas particularidades el discurso semántico de la población en su totalidad, mantiene una cohesión global enriquecida por las experiencias de su cotidianidad.

Finalmente, se determinó que el pueblo Zenú se caracteriza en su mayoría por ser personas introvertidas, que no inician turnos conversacionales, pero logran mantener, esperar y ceder tiempos, siendo actores pasivos en la interacción. Sin embargo, poseen habilidad para apoyar los temas propuestos, optando por exponer temáticas personales sobre sucesos familiares vividos. Los Zenúes hacen uso de los diferentes elementos deícticos a lo largo de su expresión (de persona, tiempo y lugar), emplean gestos naturales y espontáneos al entablar una conversación; no obstante, la mayoría, no establece contacto visual y tienen como rasgos distintivos el hablar rápido, cambiar la entonación de palabras y no emplear autocorrecciones; prevalecen las interrupciones dentro de la conversación, creando lapsos entre los intercambios comunicativos. 


\section{Referencia:}

Las referencias a otras obras son una parte

muy importante en la literatura científica;

ya que estas permiten conocer más sobre los autores y mantener vivas sus voces dentro del texto.

Acosta, V. \& Moreno, A. M. (1999). Dificultades del lenguaje en ambientes educativos. Barcelona: MASSON.

Alban, D., Calambas, L., Cobo, M. \& Ordeñez, M. (2007). Caracteriticas del lenguaje oral a nivel fonetico-fonologico del español, en niños indigenas de 7 años en la poblacion residente en el resguardo de Chimborazo. Cauca: Universidad del Cauca.Facultad ciencias de la salud.

Aleza, I. M. \& Enguita, U.J. (2010). La lengua española en América: normas y usos actuales. Valencia: Universitat de Valencia. Disponible en: http://www. uv.es/aleza/esp.am.pdf

Briceño, F. (1998). Clasificadores Maya en yucateco. En J. Calvo, \& D. Jorques, Estudios de lengua y cultura Amerindias II-Lengua Literaturas y medios (págs. 476o). España: Universidad de Valencia.Departamento teoría de los lenguaje.

Buenrostro, C. (1998). Interferencias en el español de los Chujes. En J. Perez \& D. Jorques, Estudios de lengua y cultura Amerindias II- Lengua, Literaturas y medios. (págs. 145-158). España: Universidad de Valencia.Departamento teoria de los lenguajes.

Buitrago, L., Mondragon, A., Parra, Y., \& R ivera, D. (2007). Caracterización de la postura y los procesos de respiracion y articulacion desde la interferencia fonetica en al poblacion indiegena bilingue entre 20 y 40 años de edad, del resguardo del municipio de toroto en el departamento del cauca. Cauca: universidad del cauca.Facultad ciencias de la salud.

Busto, M., Faig, V., Rafanell, L., Madrid, L. \& Martinez, G. P. (2008). Valoración del habla en niños de educacion infantil y primaria. Dialnet. Recuperado de: http://www.publicacions.ub.edu/revistes/phonica4/ documentos/576.pdf

Departamento Administrativo Nacional de Estadistica (DANE) Censo Generacional Poblacional.Recuperado de: http://www.dane.gov.co

Foley, D. E. (2004). El indigena silencioso como una produccion cultural. Obtenido de Scielo.org.ar Cuadernos de Antropologia Social: http//www.

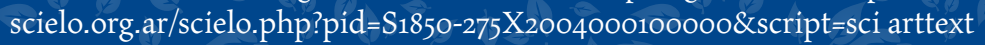

Gallego, P.J. \& Arboleda, V.L. (2004). Sintesis de la predicación en lengua Wounan. Boletín de Antropología de la Universidad de Antioquia. Disponible en internet desde: http://aprendeenlinea.udea.edu.co/revistas/ index.php/boletin/article/viewFile/6922/6337

Gallardo, R. \& Gallego, J.L. (2000). Manual de logopedia Escolar. Málaga: Aljibe. Haverkate, H. (1997). Dialogos hispanicos de Amsterdam No6. La Semiotica del dialogo, 27.
Hernandez, S., Fernandez, C., \& Baptista, L. P. (2003). Metodologia de la investigación. México: Mc Graw Hill.

Herrera, E. \& Butrageño, P. (2008). Fonologia instrumental: patrones fónicos y variación. México: Colegio de México, Centro de estudios linguisticos y literarios.

Herrera, M, Gutiérrez, C. \& Rodríguez, C. (2008). ¿Cómo detectar las dificultades del lenguaje en el nivel inicial? En "Repensar la niñez en el siglo XXI". Conferencia llevada a cabo en el X Congreso Nacional y II congreso Internacional de la Facultad de Educación Elemental y Especial de la Universidad de Cuyo, Mendoza, Argentina.

Hosfstadt, C. J. (2005). El libro de las habilidades comunicativas. España: Diaz Santos. Izquierdo, J. E. (2010). Pueblos indigenas de Colombia. Obtenido de scribd.com: http://www.scribd.com/doc/26664597/Pueblos-indigenas-de Colombia.

Luria, R. (1985). Lenguaje y pensamiento. España: Martinez Roca, S.A.

Ministerio de Cultura de la República de Colombia. (2010). Caracterizacion del pueblo Zenú. Zenú la gente de la palabra. Obtenido de mincultura.gov. co: file://C:/Users/Usucre1/Downloads/Caracterizaci\%C3\%B3n\%20del\%20 pueblo\%2oZen\%C3\%BA\%20(2).pdf

Oleron, P. (1985). El niño y al adqusicion del lenguaje. Paris: Morata S.A.

Paternina, J. D. (2002). Curiosidades Historicas del Municipio de San Andres de Sotavento-Cordoba. Barranquilla: s.n. editor.

Sánchez, T. A., Usso, T. E., Morales, V. M. \& Almendra, V. S. (2002). Interferencia Lingüística de la Lengua Castellana en la Lengua Guambiana. (Tesis pregrado). Universidad del Cauca, Popayán.

Siebold, K. (2008). Actos de habla y cortesia verbal en español y aleman. Estudio pragmalinguistico e intercultural. Frankfurt: Peter Lang GmbH Internationaler Verlag der Wissenschaften.

Trillos, A. (2001). Ayer y hoy del Caribe Colombiano en sus lenguas. Bogota: Observatorio del Caribe Colombiano.

Turbay, S. \& Jaramillo, S. (2000). Los indigenas Zenues. Geografia Humana de Colombia. Obtenido de banrepcultural.org: http://www.banrepcultural org/blaavirtual/geografia/geoco4v3/zenues.htm

Vygostky, L. (1995). Pensamiento y Lenguaje. Barcelona: Paidós. 